\title{
epiflows: an R package for risk assessment of travel-related
}

\section{spread of disease [version 1; peer review: 2 approved with}

\section{reservations]}

\section{Paula Moraga (D1) Ilaria Dorigatti (iD)2*, Zhian N. Kamvar (D)2*, Pawel Piatkowski3*, Salla E. Toikkanen4, VP Nagraj(i) 5, Christl A. Donnelly 2,6, Thibaut Jombart (i) 2,7}

\footnotetext{
${ }^{1}$ Centre for Health Informatics, Computing and Statistics (CHICAS), Lancaster Medical School, Lancaster University, Lancaster, LA1 4YW, UK

${ }^{2}$ MRC Centre for Global Infectious Disease Analysis, Department of Infectious Disease Epidemiology, School of Public Health, Imperial College, London, W2 1PG, UK

3international Institute of Molecular and Cell Biology, Warsaw, Poland

${ }^{4}$ National Institute for Health and Welfare, Helsinki, Finland

${ }^{5}$ School of Medicine, Research Computing, University of Virginia, Virginia, USA

${ }^{6}$ Department of Statistics, University of Oxford, Oxford, OX1 3LB, UK

${ }^{7}$ Department of Infectious Disease Epidemiology, London School of Hygiene and Tropical Medicine, London, UK

* Equal contributors
}

\section{V1 First published: 31 Aug 2018, 7:1374 \\ https://doi.org/10.12688/f1000research.16032.1}

Second version: 02 Aug 2019, 7:1374

https://doi.org/10.12688/f1000research.16032.2

Latest published: 12 Sep 2019, 7:1374

https://doi.org/10.12688/f1000research.16032.3

\section{Abstract}

As international travel increases worldwide, new surveillance tools are needed to help identify locations where diseases are most likely to be spread and prevention measures need to be implemented. In this paper we present epiflows, an R package for risk assessment of travelrelated spread of disease. epiflows produces estimates of the expected number of symptomatic and/or asymptomatic infections that could be introduced to other locations from the source of infection. Estimates (average and confidence intervals) of the number of infections introduced elsewhere are obtained by integrating data on the cumulative number of cases reported, population movement, length of stay and information on the distributions of the incubation and infectious periods of the disease. The package also provides tools for geocoding and visualization. We illustrate the use of epiflows by assessing the risk of travel-related spread of yellow fever cases in Southeast Brazil in December 2016 to May 2017.

Keywords

disease surveillance, outbreaks, epidemics, infectious, R, RECON

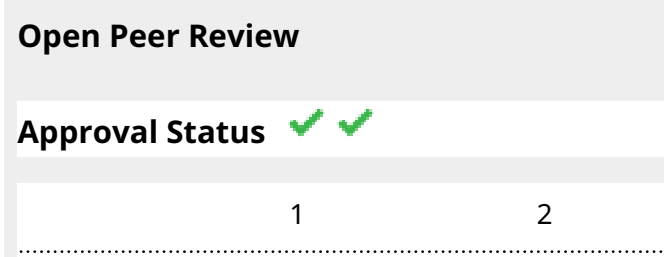

version 3

(revision)

12 Sep 2019

version 2

(revision)

02 Aug 2019

version 1

31 Aug 2018

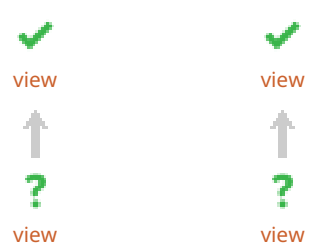

1. Noam Ross , EcoHealth Alliance, New York City, USA

2. Jon Zelner, University of Michigan, Ann Arbor, USA

Any reports and responses or comments on the article can be found at the end of the article. 
This article is included in the RPackage

gateway.

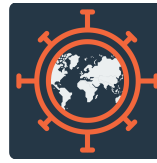

This article is included in the Emerging Diseases

and Outbreaks gateway.

Corresponding author: Paula Moraga (pm865@bath.ac.uk)

Author roles: Moraga P: Formal Analysis, Software, Writing - Original Draft Preparation, Writing - Review \& Editing; Dorigatti I: Data Curation, Formal Analysis, Methodology, Writing - Review \& Editing; Kamvar ZN: Formal Analysis, Software, Writing - Review \& Editing; Piatkowski P: Software, Writing - Review \& Editing; Toikkanen SE: Software, Writing - Review \& Editing; Nagraj V: Software, Writing Review \& Editing; Donnelly CA: Methodology, Writing - Review \& Editing; Jombart T: Software, Writing - Review \& Editing

Competing interests: No competing interests were disclosed.

Grant information: ID acknowledges research funding from the Imperial College Junior Research Fellowship. ID, CAD and TJ thank the UK Medical Research Council for Centre funding. TJ is funded by the National Institute for Health Research Health Protection Research Unit (NIHR HPRU) in Modelling Methodology at Imperial College London in partnership with Public Health England (PHE).

The funders had no role in study design, data collection and analysis, decision to publish, or preparation of the manuscript.

Copyright: @ 2018 Moraga P et al. This is an open access article distributed under the terms of the Creative Commons Attribution License, which permits unrestricted use, distribution, and reproduction in any medium, provided the original work is properly cited. Data associated with the article are available under the terms of the Creative Commons Zero "No rights reserved" data waiver (CC0 1.0 Public domain dedication).

How to cite this article: Moraga P, Dorigatti I, Kamvar ZN et al. epiflows: an R package for risk assessment of travel-related spread of disease [version 1; peer review: 2 approved with reservations] F1000Research 2018, 7:1374

https://doi.org/10.12688/f1000research.16032.1

First published: 31 Aug 2018, 7:1374 https://doi.org/10.12688/f1000research.16032.1 


\section{Introduction}

Infectious disease outbreaks cause significant suffering and mortality in the affected populations, and damage the health, social and economic well-being of the families affected by diseases as well as producing significant economic costs for local and national governments. As we have seen with Ebola and SARS, disease outbreaks can spread beyond national borders ${ }^{1}$. Travelers can acquire a disease while staying in a foreign country, and then seed new outbreaks in their home country after their return. As international travel increases worldwide, new surveillance tools are needed to help identify locations where diseases are most likely to be spread and prevention measures need to be implemented. This is essential to limit the global spread of local outbreaks.

Recently, Dorigatti et al. ${ }^{2}$ developed a method to assess the risk of travel-related international spread of disease by integrating epidemiological and travel (by air, land and water) volume. The model developed by Dorigatti et al. ${ }^{2}$ estimates the expected number of infections introduced elsewhere by taking into account population flows, lengths of stay, as well as the variability of the disease incubation and infectious periods. The method was applied to quantify the risk of spread of a recent outbreak of yellow fever in Southeast Brazil in December 2016 to May 2017, and was able to identify the countries that could have received travel-related disease cases capable of seeding local transmission.

In this paper we present epiflows, an R package that implements the method presented by Dorigatti et al. ${ }^{2}$ for risk assessment of travel-related spread of disease. Using data on population movement between the location that is source of the infection and other locations, lengths of stay, as well as information about the disease incubation and infectious period distributions, the package allows the estimation of the number of (symptomatic and/or asymptomatic) infections that could be spread to other locations together with uncertainty measures. The package also provides tools for geocoding and visualization of population flows.

The remainder of the paper is organized as follows. First, we briefly describe the modelling framework that is implemented in the epiflows package. Second, we introduce the main components of epiflows including instructions for installation and main functions. Third, we illustrate the use of the package via the assessment of the risk of travel-related spread of yellow fever cases due to population flows between Southeast Brazil and other countries in December 2016 to May 2017. Specifically, we discuss the data required and show how to perform the statistical analyses, how to interpret the results, and the visualization options. Finally, the conclusions are presented.

\section{Model}

In this Section we explain the modelling framework presented in 2 for estimating the expected number of infections departing from one infectious location during the incubation or infectious periods. These cases comprise exportations and importations. Exportations refer to the infected residents of the infectious location (i.e. location with sustained disease transmission) that travel to other locations. Importations (also referred to as returning travelers) are people that are infected during a temporary stay in the infectious location and then return to their home location. The following Sections describe how to model exportations and importations to produce the total number of expected cases that could be spread to other locations together with uncertainty measures.

\section{Exportations}

Let $C_{S, W}$ denote the cumulative number of infections in location $S$ in time window $W$. Here, $W$ denotes the temporal window between the first and the last disease case in location $S$. Note that Dorigatti et al. ${ }^{2}$ calculated $C_{S, W}$ by multiplying the number of confirmed and reported yellow fever cases by 10 to account for underreporting of asymptomatic and mild yellow fever cases.

Let pop $_{S}$ be the resident population of the infectious location $S$, and $T_{S, D}^{W}$ the number of residents of location $S$ travelling to location $D$ in time window $W$. The per capita probability that a resident from the infectious location travelled to other location $D$ during the time window $W$ is given by

$$
p_{D}=\frac{T_{S, D}^{W}}{\text { pop }_{S}} .
$$

The method assumes that the incubation period $T_{E}$ and the infectious period $T_{I}$ follow specific probability distributions. Using these, we can calculate the probability $p_{i}$ that an infection incubated or is infectious in time window $W$ as

$$
p_{i}=\operatorname{minimum}\left(\frac{T_{E}+T_{I}}{W}, 1\right)
$$


Finally, the number of residents of the infectious location $S$ that are infected and travel abroad during their incubation or infectious period during the time window $W$ can be calculated as

$$
E_{S, D}=C_{S, W} \times p_{D} \times p_{i}
$$

That is, $E_{S, D}$ is a product of the cumulative number of infections in location $S$ in time window $W$, the per capita probability that a resident of $S$ travels to location $D$, and the probability that an infection incubated or is infectious in time window $W$.

Note here that if travel data are expressed annually $\left(T_{S, D}^{A}\right)$ instead of in the time window $W$, travel data in the time window can be obtained as $T_{S, D}^{W}=\left(T_{S, D}^{A} \times W\right) / 365$.

Importations

Let $T_{O, S}^{W}$ be the number of travelers visiting location $S$ from location $O$ in time window $W$, and let $L_{O}$ denote the average length of stay. The per capita risk of infection of travelers visiting location $S$ during their stay can be calculated as

$$
\lambda_{S}=\frac{C_{S, W} \times L_{O}}{\text { pop }_{S} \times W} .
$$

The probability of returning to the home location while incubating or infectious is given by

$$
p_{l}=\operatorname{minimum}\left(\frac{T_{E}+T_{I}}{L_{O}}, 1\right) .
$$

Finally, the expected number of travelers infected during their stay in the infectious location and returning to their home location before the end of the infectious period can be calculated as the product of the number of travelers, the per capita risk of infection and the probability of returning home while incubating or infectious,

$$
I_{S, O}=T_{O, S}^{W} \times \lambda_{\mathrm{S}} \times p_{l}
$$

Note that, similarly to exportations, if travel data are expressed annually $\left(T_{O S}^{A}\right)$ instead of in the time window $W$, travel data in the time window can be obtained as $T_{O, S}^{W}=\left(T_{O, S}^{A} \times W\right) / 365$.

\section{Total number of exportations and importations}

Finally, the expected number of infections departing from the infectious location $S$ to location $O$ during the incubation or infectious periods can be computed as the sum of the number of infected residents of $S$ travelling during their incubation or infectious periods, and the travelers from abroad that are infected during their stay in $S$ and return to their origin location before the end of the infectious period. That is,

$$
T_{S, O}=E_{S, O}+I_{S, O}
$$

Average estimates and the relative uncertainty are calculated by taking into account the variability of the incubation and infectious periods. Specifically, the method samples a large number of times from the incubation and infectious distributions, which produces a full distribution for $p_{i}$ (the probability that a disease case is incubated or infectious in the time window considered) and $p_{l}$ (the probability of returning to the home location while incubating or infectious). This, in turn, creates variability in exportations $E_{S, O}$ and importations $I_{S, O}$, and finally in the total number of infections introduced in location $\mathrm{O}, T_{S, O}$.

\section{Methods}

Implementation

The R package epiflows [17] is hosted in the Comprehensive R Archive Network (CRAN) which is the main repository for $\mathrm{R}$ packages: http://CRAN.R-project.org/package=epiflows. Users can install epiflows in R by executing the following code:

install.packages ("epiflows")

There is also a development version from GitHub which can be accessed at https://github.com/reconhub/ epiflows. This version of the package may contain new features which are not incorporated in the version on CRAN yet but may be useful for some users. GitHub also includes issue tracking where users can note problems 
or suggestions for improvements. This development version from GitHub can be installed by using the install_github () function from the R package devtools $\mathrm{s}^{3}$ :

install.packages ("devtools")

library ("devtools")

install_github("reconhub/epiflows")

When installing epiflows, other R packages which epiflows depends on are also automatically installed. These packages include $\mathrm{sp}^{4}$ for manipulating spatial objects; geosphere $\mathrm{e}^{5}$ for calculating distances between locations; and leaf $l$ et ${ }^{6}$ for visualization.

\section{Operation}

The main function of the package is estimate_risk_spread() which calculates the mean and 95\% confidence intervals of the number of cases spread to different locations from an infectious location. It is also possible to use this function to produce a data frame with all simulations (not just the mean and $95 \%$ confidence intervals that is computed from the simulations). This permits the user to aggregate the estimates and calculate confidence intervals with different levels using single simulations. To execute this function the following information is needed:

- population of the infectious location,

- number of infections in the infectious location, and the first and last dates of reported cases,

- number of travelers between the infectious location and other locations,

- average length of stay of travelers from other locations visiting the infectious location,

- distributions of the incubation and infectious periods,

- number of simulations to be drawn from the incubation and infectious period distributions,

- logical value indicating whether the returned object should be a data frame with all simulations, or a data frame with the mean and lower and upper limits of a $95 \%$ confidence interval of the number of infections spread to each location.

Other useful functions are plot() which produces visualizations of population flows between locations, and add coordinates () which finds the coordinates of the locations.

\section{Use cases}

In this Section we provide an example on how to use epiflows to calculate the number of yellow fever cases spreading from south-east Brazil to other countries due to human movement. We show how to define the arguments of the estimate risk spread() function, interpret the results, and make visualizations with the population flows.

\section{Data}

We use the data YF Brazil which is contained in the epiflows package as data (YF Brazil). YF Brazil is a list containing the population size, the assumed number of yellow fever infections, $\overline{\text { dates }}$ of first and last case reporting, number of travelers and length of stay for the states of Espirito Santo, Minas Gerais, Rio de Janeiro, Sao Paulo, and for the whole region of Southeast Brazil (which comprises the four states of Espirito Santo, Minas Gerais, Rio de Janeiro and Sao Paulo) in the period December 2016 to May 2017 [15], [16].

Following Dorigatti et al. ${ }^{2}$, the total number of yellow fever infections in each of the Brazilian states was calculated by multiplying the cumulative number of confirmed yellow fever cases reported in 7 by 10 to account for underreporting of asymptomatic and mild yellow fever cases. The dates of first and last case reported in each state were derived as described by Dorigatti et $a .^{2}$. Population data were obtained from the Brazilian Institute of Geography and Statistics website ${ }^{8}$. YF Brazil also contains the number of travelers in the specified time window between the states of Espirito Santo, Minas Gerais, Rio de Janeiro, Sao Paulo (and the whole Southeast Brazilian region) and other countries. These estimates were obtained from World Tourism Organization data on the volume of air, land and water border crossings for Brazil for the year $2015^{\circ}$, having assumed that travelers were distributed across the Brazilian states according to the relative population density and having accounted for information on the monthly distribution of tourism and on the average duration of stay of international visitors to Brazil $^{10}$, as detailed in 2 . 
YF_Brazil\$states is a data frame that contains, for each Brazilian state considered in our example, the code (location code), the population (location population), the number of assumed infections in the time window (num_cases_time_window), and the dates of the first and last case reported (first_date_ cases and last_date_cases, respectively).

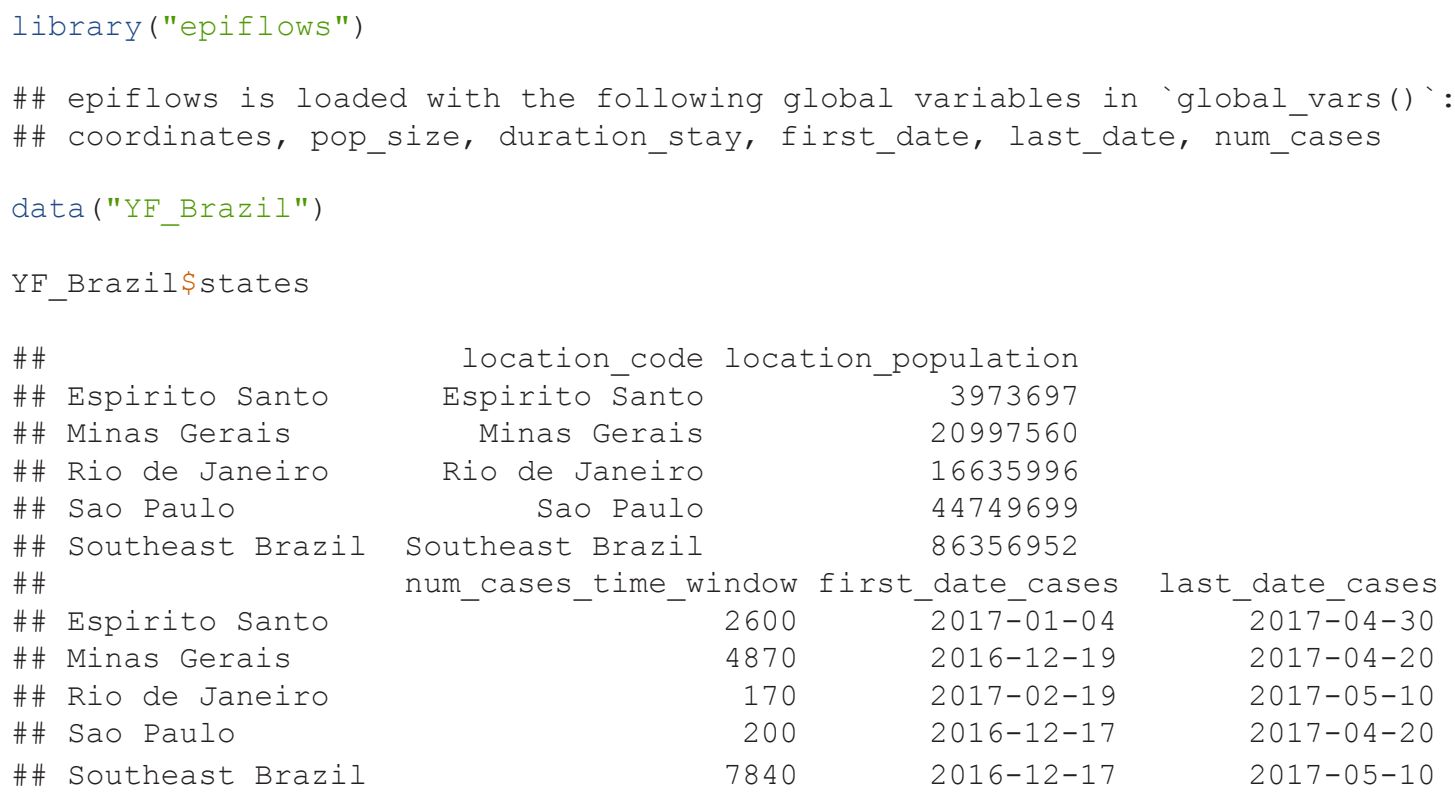

YF_Brazil $\$ T \_D$ represents $T_{S, D}^{W}$ and contains the number of travelers from each of the Brazilian states to other countries. Y Y to each of the Brazilian states.

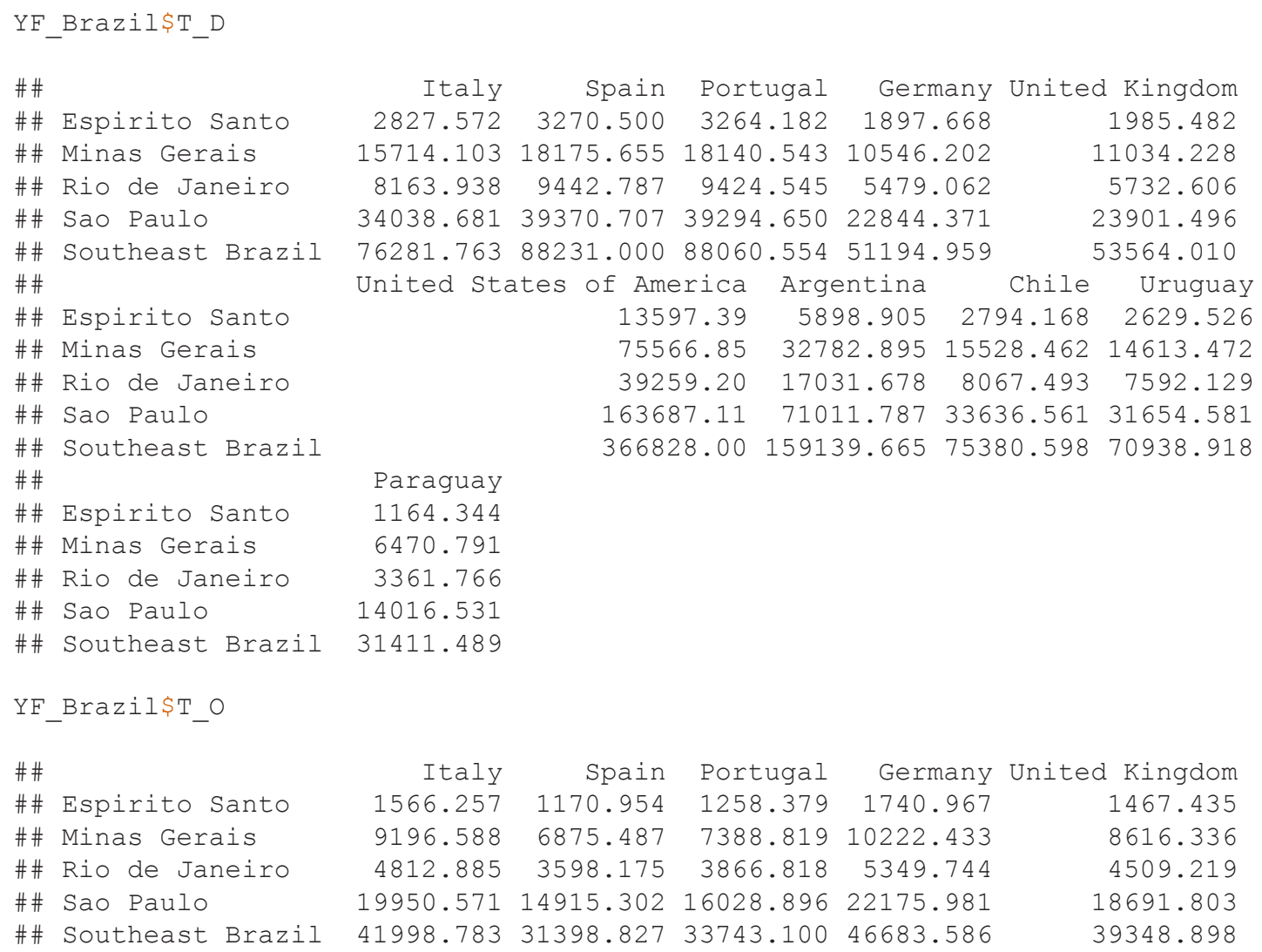

$\begin{array}{rrrrr}\text { Italy } & \text { Spain } & \text { Portugal } & \text { Germany } & \text { United Kingdom } \\ 1566.257 & 1170.954 & 1258.379 & 1740.967 & 1467.435 \\ 9196.588 & 6875.487 & 7388.819 & 10222.433 & 8616.336 \\ 4812.885 & 3598.175 & 3866.818 & 5349.744 & 4509.219 \\ 19950.571 & 14915.302 & 16028.896 & 22175.981 & 18691.803 \\ 41998.783 & 31398.827 & 33743.100 & 46683.586 & 39348.898\end{array}$




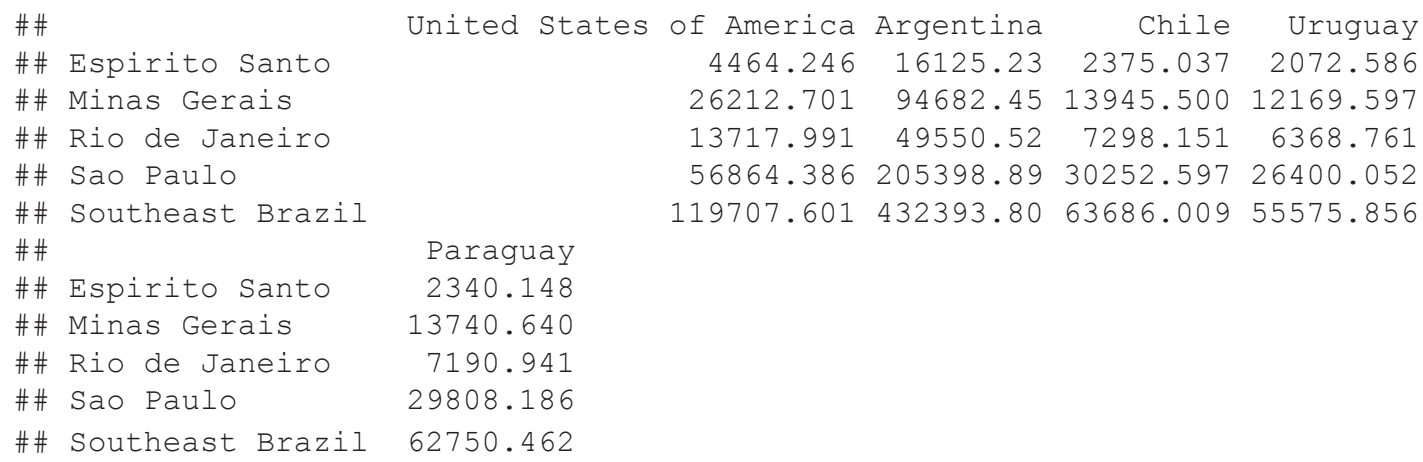

Finally, YF_Brazil\$length_of_stay is a vector with the lengths of stay (in days) of the travelers visiting Brazil from other countries.

$\begin{array}{lrrr}\text { YF_Brazil\$length_of_stay } & & \\ \text { \#\# } & \text { Italy } & & \\ \# \# & 30.1 & \text { Spain } & \text { Portugal } \\ \# \# & 27.2 & 27.2 \\ \# \# & \text { Germany } & \text { United Kingdom United States of America } \\ \# \# & 22.3 & 19.5 & 18.5 \\ \# \# & \text { Argentina } & \text { Chile } & \text { Uruguay } \\ \# \# & 10.9 & 10.3 & 8.0 \\ \# \# & \text { Paraguay } & & \end{array}$

The epiflows object

To aid in data organization between flows and metadata, we have implemented the "epiflows" object. This inherits the "epicontacts" object from the epicontacts package" ${ }^{11}$, storing three elements:

1. flows - a data frame defining the number of cases flowing from one location to another

2. locations - a data frame listing the locations present in f lows and relevant metadata.

3. vars - a dictionary mapping column names in locations to known global variables defined in global_vars (). These global variables are used as default values in estimate_risk_spread ().

An epiflows object can be created with the make epiflows() function by providing a data frame flows with the number of travelers between locations, a data frame locations with information about the locations, and the names of the columns of data frame locations indicating the name of each variable.

In the data frame flows each row represents the number of travelers from one location to the next. flows has at least three columns: columns from and to indicating where the flow starts and ends, respectively, and column $\mathrm{n}$ indicating the number of travelers that are in the flow. We can create a data frame YF flows with the population flows of the Brazil data as follows.

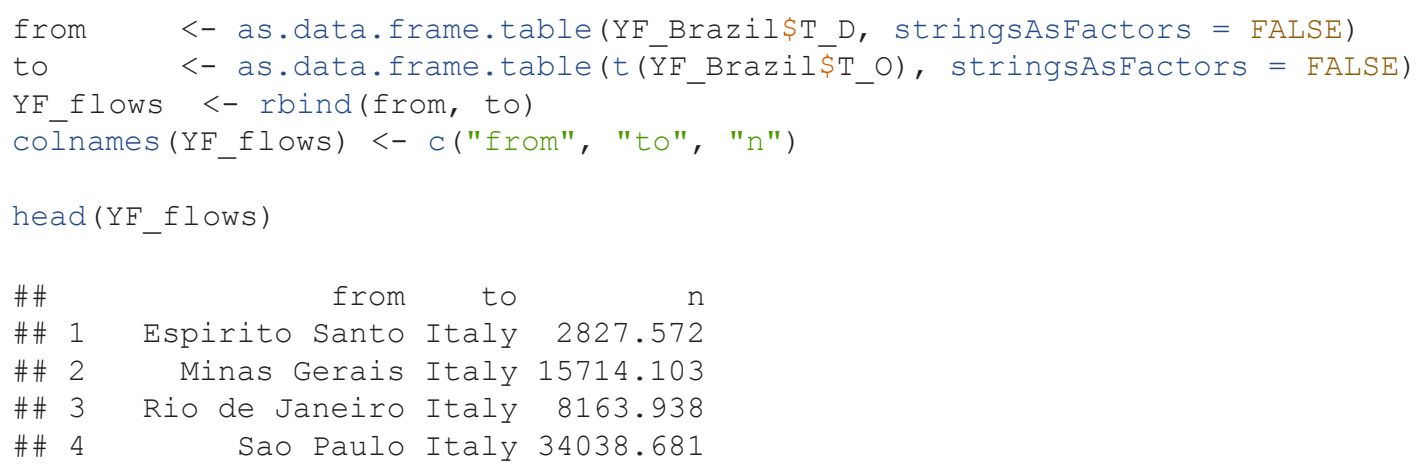


In data frame locations each row represents a location, and columns specify useful information about the locations such as ID, population, number of cases, dates and length of stay. locations must contain at least one column specifying the location ID used in the flows data frame. We can create the data frame $Y F$ locations by combining the data frame YF Brazil\$states containing the Brazil states information, and the vector YF Brazil\$length_of stay containing the lengths of stay.

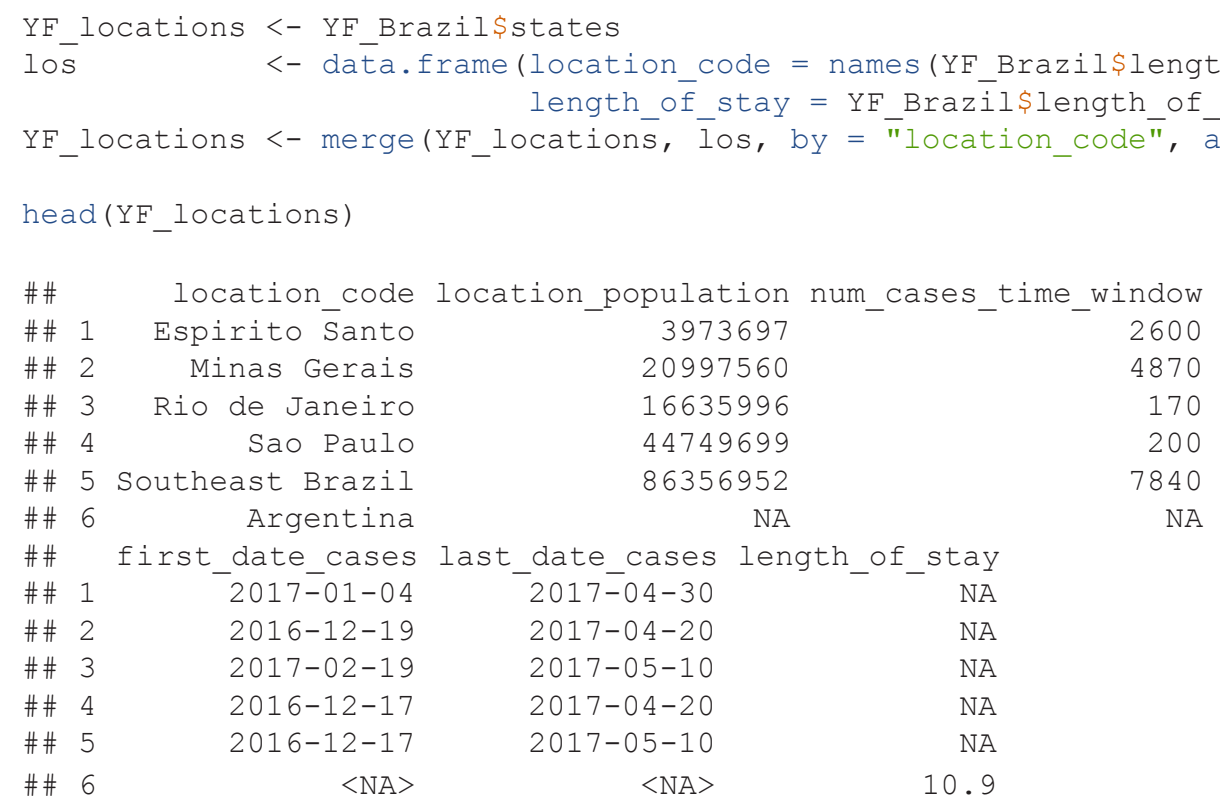

Then, we can create an epiflows object called Brazil_epiflows as follows.

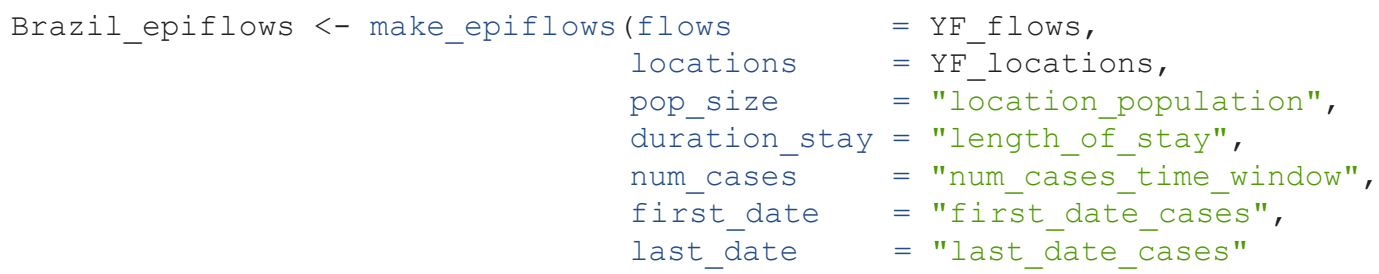

)

Arguments of the estimate_risk_spread () function

The arguments that need to be specified in estimate_risk_spread() to calculate the cases or infections introduced in other countries are as follows. The first argument is an epiflows object containing the number of travelers between locations, the population size, the number of cases, and the first and last dates of reporting in the infectious location, and the average length of stay in days of travelers from other locations visiting the infectious location.

The second argument of estimate risk spread() is location code which is a character string denoting the infectious location code. We also need to specify the incubation and infectious period distributions. Specifically, we need to provide functions with a single argument $\mathrm{n}$ that generate $\mathrm{n}$ random incubation and infectious periods. In this example, we assume that the incubation period $T_{E}$ is log-normally distributed with mean 4.6 days and variance 2.7 days, and that the infectious period $T_{I}$ is normally distributed with mean 4.5 days and variance 0.6 days. We can define functions incubation () and infectious () as

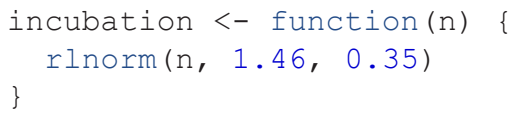




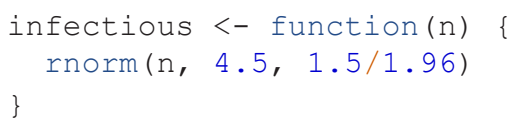

Argument num_sim is the number of simulations to be drawn from the incubation and infectious period distributions. It is recommended to use at least 1,000 simulations. The last argument of estimate_risk_spread () is return all simulations. This is a logical value indicating whether the returned object should be a data frame with all simulations (return all simulations = TRUE), or a data frame with the mean and lower and upper limits of a $95 \%$ confidence interval of the number of infections spread to each location (return all simulations = FALSE).

\section{Execution of the estimate_risk_spread () function}

Once we have constructed the objects needed to call estimate_risk_spread() we can execute the function and obtain the estimated mean number of cases spread to each country and the $95 \%$ confidence intervals. The code to calculate the cases spread from Espirito Santo is the following:

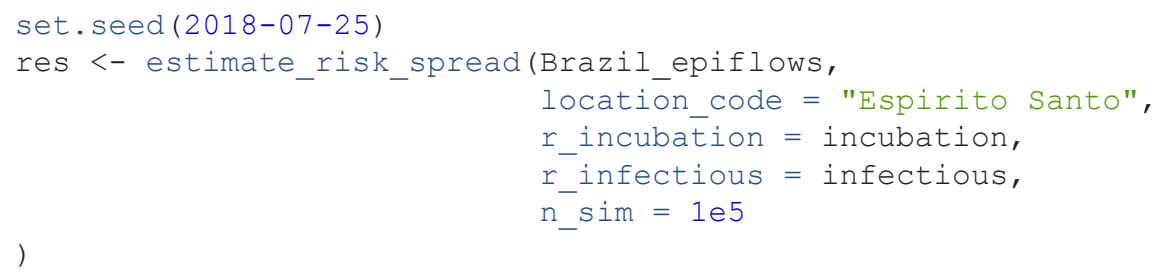

The results returned by estimate_risk_spread() are stored in the res object. This is a data frame with the columns mean cases indicating the mean number of cases spread to each location, and lower limit $95 \mathrm{CI}$ and upper limit 95CI indicating the lower and upper limits of 95\% confidence intervals. The result object is shown below.

\begin{tabular}{|c|c|c|c|c|}
\hline \#\# & & mean_cases & lower_limit_95CI & upper_limit_95CI \\
\hline & Italy & $0.2 \overline{2} 33656$ & $0.15 \overline{2} 0966$ & $0.30 \overline{7} 8136$ \\
\hline \#\# & Spain & 0.2255171 & 0.1537452 & 0.3126801 \\
\hline \#\# & Portugal & 0.2317019 & 0.1565528 & 0.3383112 \\
\hline \#\# & Germany & 0.1864162 & 0.1259548 & 0.2721890 \\
\hline \#\# & United Kingdom & 0.1613418 & 0.1195261 & 0.2089475 \\
\hline \#\# & United States of America & 0.9253419 & 0.6252207 & 1.3511047 \\
\hline \#\# & Argentina & 1.1283506 & 0.7623865 & 1.6475205 \\
\hline \#\# & Chile & 0.2648277 & 0.1789370 & 0.3866836 \\
\hline \#\# & Uruguay & 0.2408942 & 0.1627681 & 0.3517426 \\
\hline \#\# & Paraguay & 0.1619724 & 0.1213114 & 0.1926966 \\
\hline
\end{tabular}

We can plot the results with ggplot () as follows (Figure 1).

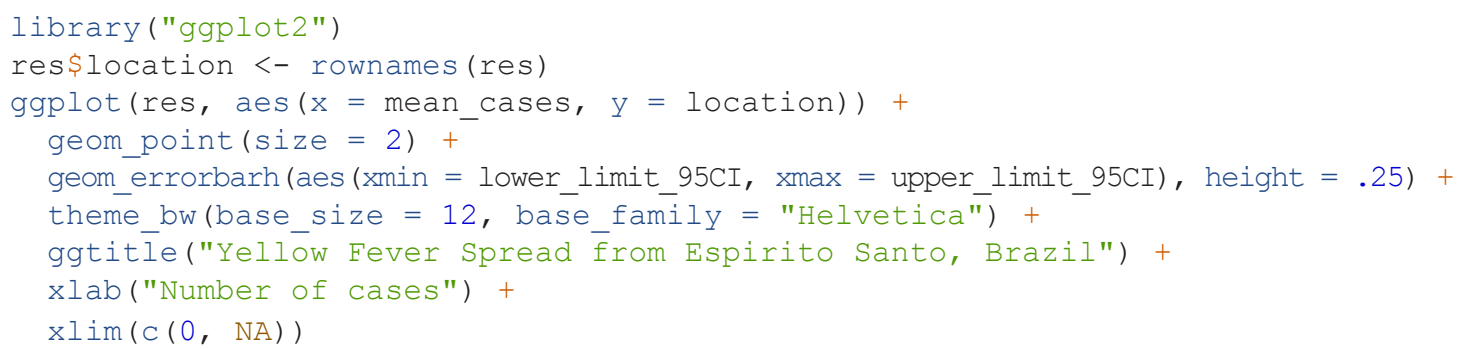




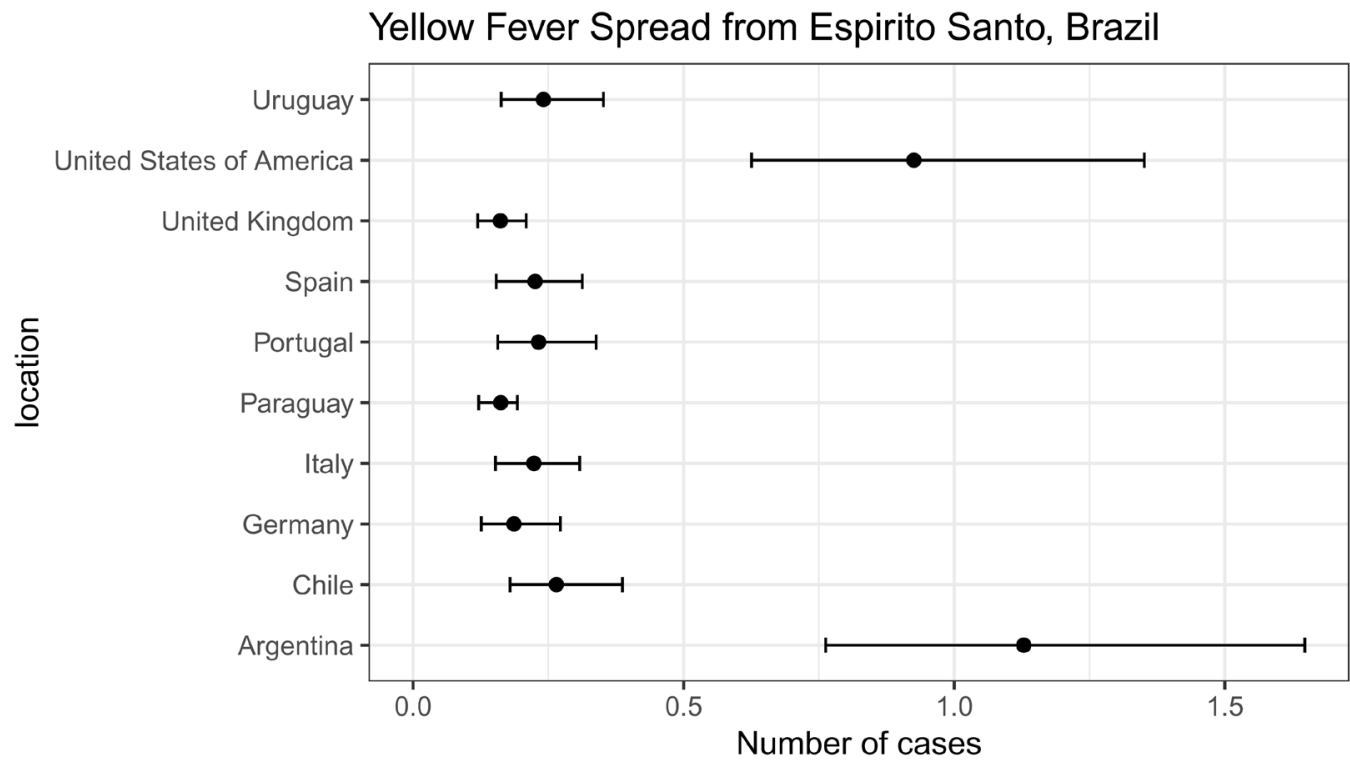

Figure 1. Mean number of yellow fever cases and $95 \% \mathrm{Cl}$ spread from Espirito Santo to other locations.

Note that if we set return_all_simulations equal to TRUE, the result object res will be a data frame with all simulations.

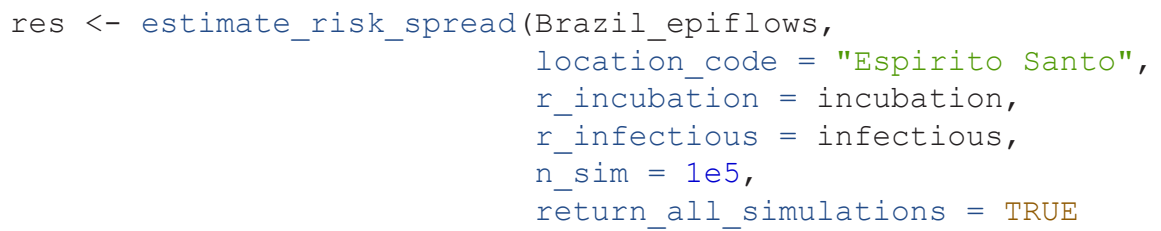

Using res, we can calculate the mean and $95 \%$ confidence intervals as follows.

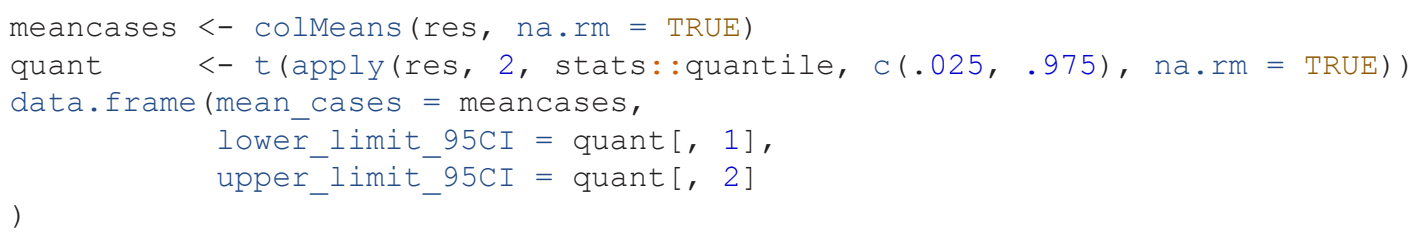




$\begin{array}{lrrr}\text { \#\# } & \text { mean_cases } & \text { lower_limit_95CI } & \text { upper_limit_95CI } \\ \text { \#\# Italy } & 0.2233975 & 0.1522848 & 0.3081296 \\ \text { \#\# Spain } & 0.2255621 & 0.1539354 & 0.3130456 \\ \text { \#\# Portugal } & 0.2317602 & 0.1567465 & 0.3388166 \\ \text { \#\# Germany } & 0.1864633 & 0.1261107 & 0.2725956 \\ \text { \#\# United Kingdom } & 0.1613646 & 0.1196739 & 0.2091694 \\ \text { \#\# United States of America } & 0.9255753 & 0.6259942 & 1.3531231 \\ \text { \#\# Argentina } & 1.1286353 & 0.7633297 & 1.6499817 \\ \text { \#\# Chile } & 0.2648933 & 0.1791584 & 0.3872613 \\ \text { \#\# Uruguay } & 0.2409532 & 0.1629695 & 0.3522681 \\ \text { \#\# Paraguay } & 0.1619776 & 0.1214615 & 0.1928268\end{array}$

Visualize population flows

We can visualize flows of people travelling between locations using plot () and passing as first parameter an epiflows object containing the population flows, and as second parameter the type of plot we wish to produce. Population flows can be displayed on an interactive map, as a network or as a grid between origins and destinations as described in the following sections.

Flows displayed on an interactive map

We can visualize population flows on an interactive map using plot () with the parameter type = "map". For this option to work, the epiflows object needs to include the longitude and latitude of the locations in decimal degree format. If coordinates are known, they can be added to the epiflows object using the add_coordinates () function from the epiflows package. In our example, the longitude and latitude data are in the data frame $Y F$ coordinates.

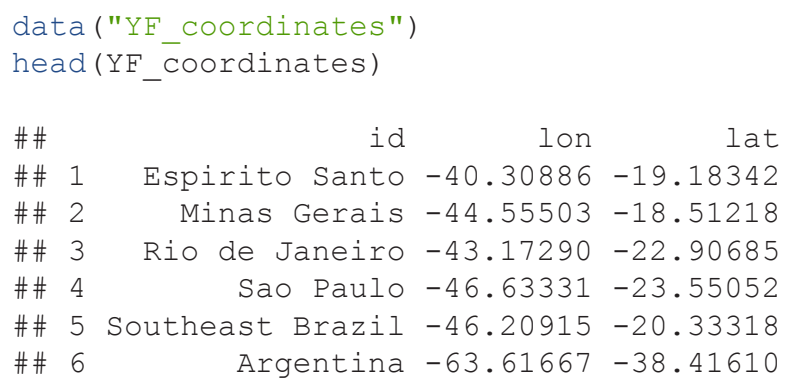

They can be added as follows.

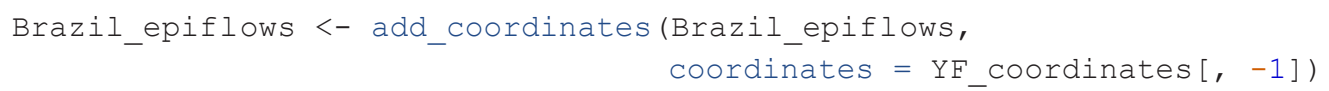

If coordinates are unknown, we may resort to one of the freely available tools for geocoding. For example, we can use the geocode () function from the ggmap package ${ }^{12}$. This function finds the latitude and longitude of a given location using either the Data Science Toolkit or Google Maps. We can also use add coordinates () which uses geocode ( ) to find the coordinates and directly add them to the epiflows object as follows.

Brazil_epiflows <- add_coordinates (Brazil epiflows, overwrite = TRUE)

Once we have assigned coordinates to the epiflows object, we can use plot () with type = "map" to visualize the population flows between locations in an interactive map (Figure 2).

plot(Brazil_epiflows, type = "map")

The produced map can be zoomed and permits an easy examination of flows. plot() uses the gcIntermediate() function from the geosphere package ${ }^{5}$ to obtain the great circle arcs between locations, and then uses the leaflet package ${ }^{6}$ to create an interactive map with the connection lines. The connection lines are coloured according to flow volume, and as the mouse passes over the lines, lines highlight and information about connections is shown. We can also include parameters to specify a title, the center of the map or a color palette. An interactive version of this visualization is shown here: https://www.repidemicsconsortium.org/ epiflows/articles/introduction.html\#introduction-epiflows-map. 


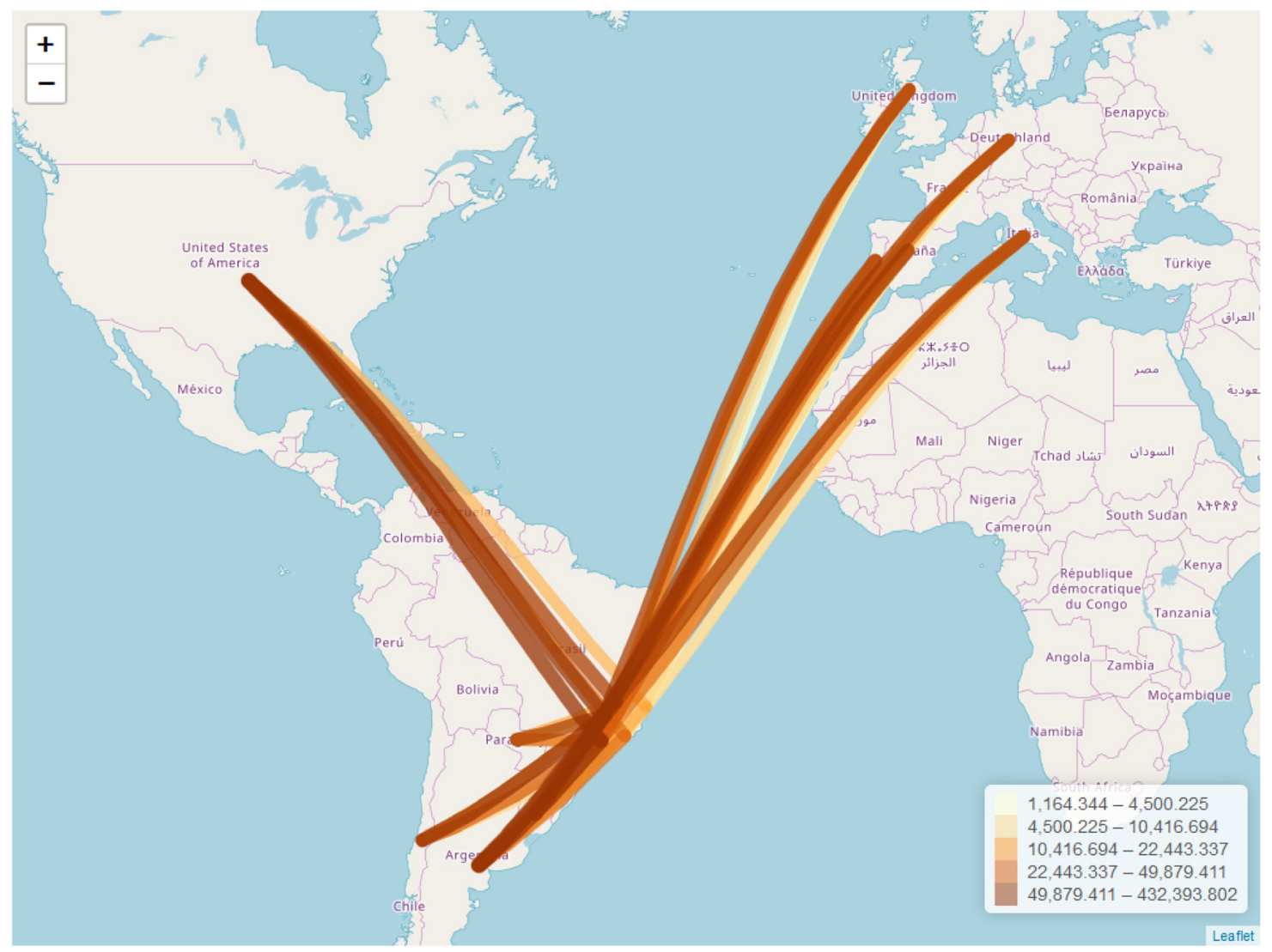

Figure 2. Population flows between Brazil states and other locations plotted using type = "map".

Flows displayed as a network

Population flows can also be displayed as a dynamic network using plot ( ) with type = "network" (Figure 3).

plot(Brazil epiflows, type = "network")

This option uses the package visNetwork $\mathrm{k}^{13}$ to show the locations as nodes of a network and connections between them representing population flows. This plot is interactive and it is possible to highlight a given location and examine its population flows, as well as its population, number of cases, dates and length of stay. This type of plot can be used when coordinates of locations are missing. An interactive version of this plot can be viewed here: https://www.repidemicsconsortium.org/epiflows/articles/introduction.html\#introduction-epiflows-vis.

Flows displayed as a grid between origins and destinations

Finally, population flows can also be shown as a grid between locations with the option type = "grid" (Figure 4).

plot(Brazil_epiflows, type = "grid")

This plot shows flows between locations as points by positioning origins and destination in $\mathrm{y}$ and $\mathrm{x}$ axes, respectively. When using this option, additional arguments can be passed to set the size, color or shape of the points as in function geom point () of package ggplot $2^{14}$. As the network plot, the grid plot can be used when coordinates of locations are missing. 


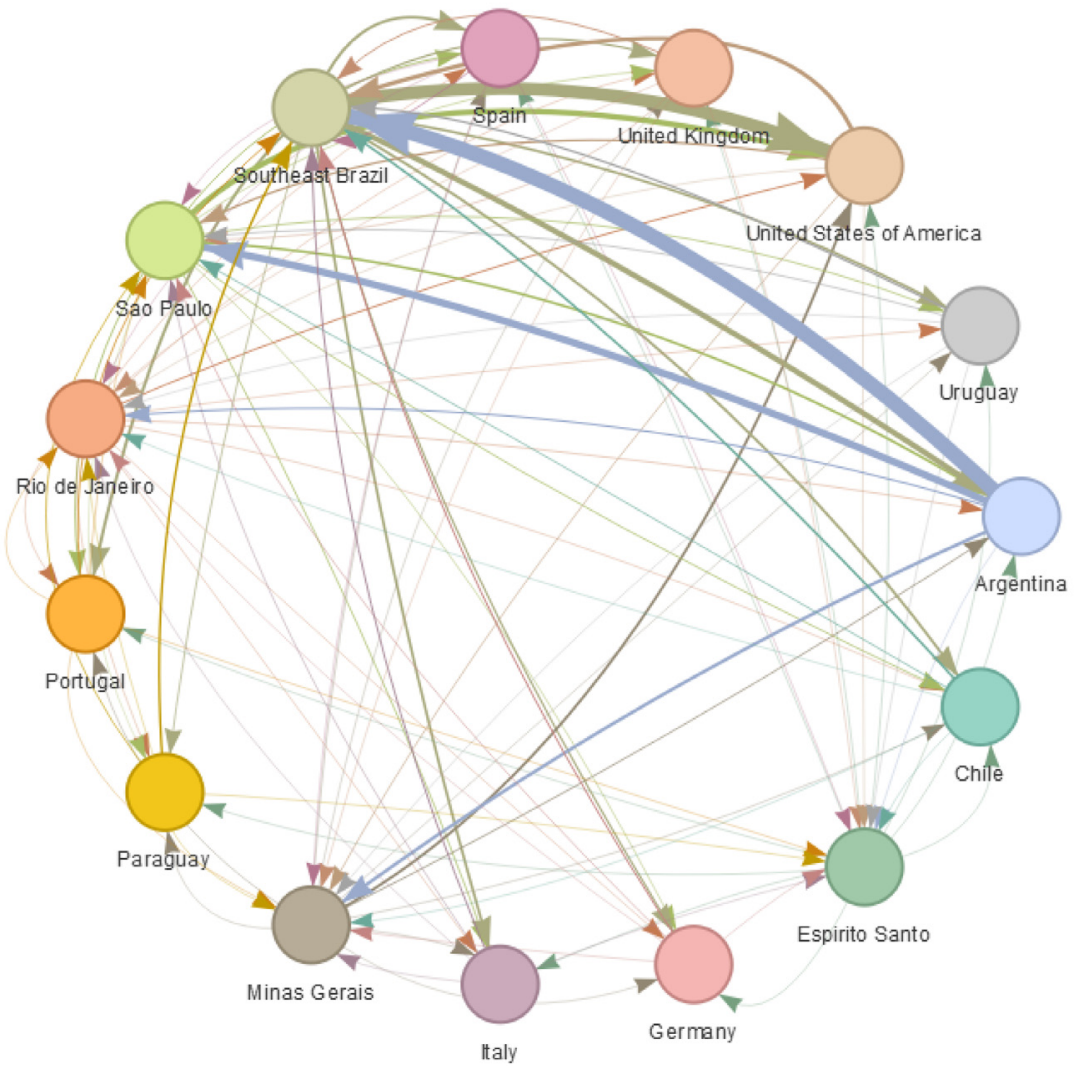

Figure 3. Population flows between Brazil states and other locations plotted using type = "network".

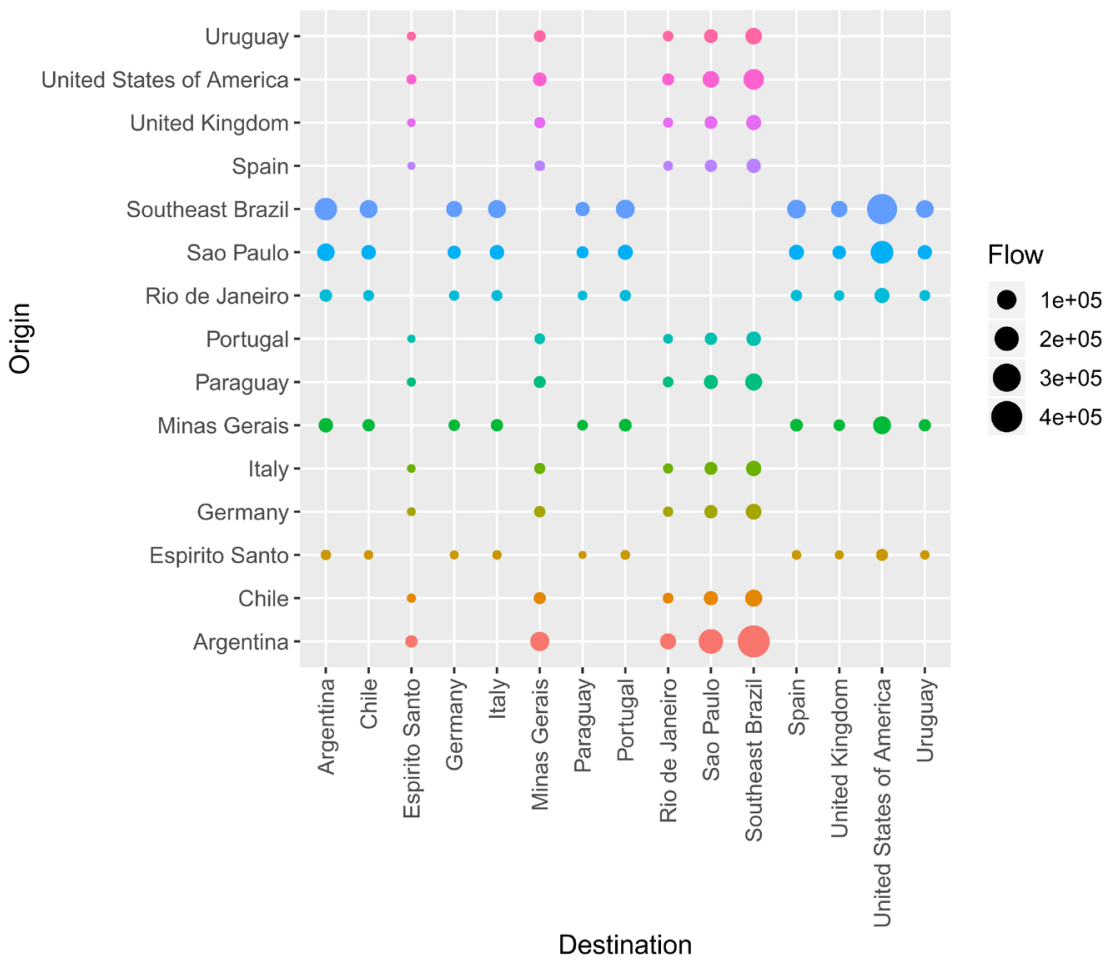

Figure 4. Population flows between Brazil states and other locations plotted using type = "grid". 


\section{Dataset 1. Arrivals of non-resident tourists at Brazilian national borders by country of residence}

https://doi.org/10.5256/f1000research.16032.d215763

Annual volumes of air, land and water border crossings for Brazil relative to inbound tourism from years 2011 to 2015 obtained from the World Tourism Organisation (UNWTO).

\section{Dataset 2. Trips abroad by Brazilian resident visitors to countries of destination}

https://doi.org/10.5256/f1000research.16032.d215765

Annual volumes of air, land and water border crossings for Brazil relative to outbound tourism from years 2011 to 2015

obtained from the World Tourism Organisation (UNWTO).

\section{Summary}

In this article we have presented the epiflows package for risk assessment of travel-related spread of disease. This package allows the estimation of the expected number of infections that could be introduced to other locations from the source of infection by integrating data on the number of cases reported, population movement, length of stay and information on the distributions of the incubation and infectious periods of the disease. The package also provides tools for geocoding and visualization which facilitate the interpretation of the results.

First, we presented how to estimate exportations, importations and total number of infections using the modelling framework introduced by Dorigatti et al..$^{2}$. Then, we demonstrated the use of the package by assessing the risk of travel-related spread of yellow fever cases in Southeast Brazil in December 2016 to May 2017. Specifically, we have shown how to construct an epiflows object containing population flows and information about locations, and how to use the function estimate_risk_spread () to obtain the average and confidence intervals of the estimated number of infections introduced elsewhere. Finally, we have shown how to visualize the results and produce maps of the population flows.

International travel has an important role in the spread of infectious diseases across national borders. We think the epiflows package represents a useful tool for disease surveillance that can help public health officials identify locations where diseases are most likely to spread and prevention measures are most needed.

\section{Data availability}

Dataset 1. Arrivals of non-resident tourists at Brazilian national borders by country of residence. Annual volumes of air, land and water border crossings for Brazil relative to inbound tourism from years 2011 to 2015 obtained from the World Tourism Organisation. https://doi.org/10.5256/f1000research.16032.d215763'15.

Dataset 2. Trips abroad by Brazilian resident visitors to countries of destination. Annual volumes of air, land and water border crossings for Brazil relative to outbound tourism from years 2011 to 2015 obtained from the World Tourism Organisation. https://doi.org/10.5256/f1000research.16032.d215765 ${ }^{16}$.

\section{Software availability}

1. Dedicated website for epiflows, including installation guidelines and documentation: https://www.repidemicsconsortium.org/epiflows

2. Software available from: https://cran.r-project.org/package=epiflows

3. Source code available from: https://github.com/reconhub/epiflows

4. Archived source code at time of publication: http://doi.org/10.5281/zenodo.1401806 ${ }^{17}$.

5. Software license: MIT License

Author contributions

PM, ZNK, PP and TJ developed the R package.

ST and VPN contributed to the R package.

ID and CAD developed the methods.

ID contributed data. 
PM, ID and ZNK analysed the data.

PM wrote the first draft of the manuscript.

All authors read and approved the final manuscript.

Grant information

ID acknowledges research funding from the Imperial College Junior Research Fellowship. ID, CAD and TJ thank the UK Medical Research Council for Centre funding. TJ is funded by the National Institute for Health Research Health Protection Research Unit (NIHR HPRU) in Modelling Methodology at Imperial College London in partnership with Public Health England (PHE).

The funders had no role in study design, data collection and analysis, decision to publish, or preparation of the manuscript.

\section{Acknowledgments}

We would like to thank the World Tourism Organisation for the permission to make public use of the border crossing data compiled by UNTWO and purchased on ${ }^{9}$. We would also like to thank the RECON consortium for establishing the research platform where this collaborative work was implemented.

1. Heymann DL, Chen L, Takemi K, et al.: Global health security: the wider lessons from the west African Ebola virus disease epidemic. Lancet. 2015; 385(9980): 1884-1901.

PubMed Abstract | Publisher Full Text | Free Full Text

2. Dorigatti I, Hamlet A, Aguas R, et al.: International risk of yellow fever spread from the ongoing outbreak in Brazil, December 2016 to May 2017. Euro Surveill. 2017; 22(28): pii: 30572. PubMed Abstract | Publisher Full Text | Free Full Text

3. Wickham H, Chang W: devtools: Tools to Make Developing R Packages Easier. R package version 1.13.3. 2017. Reference Source

4. Pebesma EJ, Bivand RS: Classes and methods for spatial data in R. $R$ News. 2005; 5(2): 9-13.

Reference Source

5. Hijmans RJ: geosphere: Spherical Trigonometry. R package version 1.5-5. 2016

Reference Source

6. Cheng J, Karambelkar B, Xie Y: leaflet: Create Interactive Web Maps with the JavaScript 'Leaflet' Library. R package version 1.1.0. 2017. Reference Source

7. Brasilia: Ministério da Saúde. Portuguese: Monitoramento dos casos e óbitos de febre amarela no Brasil, informe n. 43/2017. [Monitoring of the cases and deaths due to yellow fever in Brazil, update n. 43/2017], 2017.

Reference Source

8. Rio de Janeiro: Instituto Brasileiro de Geografia e Estatística (IBGE): Estimativas populacionais para os municípios e para as Unidades da Federação brasileiros em 01.07.2016. [Population estimates for the municipalities and for the Brazilian Federal Units on 1 July 2016.], 2016.

Reference Source
9. Madrid: UN World Tourism Organization (UNWTO): Yearbook of tourism statistics dataset. 2016. Reference Source

10. Ministério do Turismo: Estudo da Demanda Turística Internacional 2015. Study of the international tourist demand 2015, 2017. Reference Source

11. Nagraj VP, Randhawa N, Campbell F, et al.: epicontacts: Handling, visualisation and analysis of epidemiological contacts [version 1; referees: 1 approved, 1 approved with reservations]. F1000Research. 2018; 7(566). Publisher Full Text

12. Kahle D, Wickham H: ggmap: Spatial visualization with ggplot2. $R$ J. 2013; 5(1): 144-161. Reference Source

13. Almende BV, Thieurmel B, Robert T: visNetwork: Network Visualization using 'vis.js' Library. R package version 2.0.4. 2018. Reference Source

14. Wickham H: ggplot2: Elegant Graphics for Data Analysis. Springer-Verlag New York, 2009; ISBN 978-0-387-98140-6. Reference Source

15. Moraga P, Dorigatti I, Kamvar ZN, et al.: Dataset 1 in: epiflows: an $R$ package for risk assessment of travel-related spread of disease. F1000Research. 2018 http://www.doi.org/10.5256/f1000research.16032.d215763

16. Moraga P, Dorigatti I, Kamvar ZN, et al.: Dataset $\mathbf{2}$ in: epiflows: an $R$ package for risk assessment of travel-related spread of disease. F1000Research. 2018.

http://www.doi.org/10.5256/f1000research.16032.d215765

17. Kamvar ZN, Piątkowski P, Jombart T, et al.: reconhub/epiflows: Version 0.2.1: First zenodo release (Version v0.2.1). Zenodo. 2018 http://www.doi.org/10.5281/zenodo.1401806 


\title{
Open Peer Review
}

\section{Current Peer Review Status: ? ?}

\section{Version 1}

Reviewer Report 18 October 2018

https://doi.org/10.5256/f1000research.17509.r38978

(C) 2018 Zelner J. This is an open access peer review report distributed under the terms of the Creative Commons Attribution License, which permits unrestricted use, distribution, and reproduction in any medium, provided the original work is properly cited.

\author{
Jon Zelner \\ Department of Epidemiology, University of Michigan, Ann Arbor, MI, USA
}

In this paper, the authors have presented and provided use-case examples for an $\mathrm{R}$ package that allows the estimation of the number of infections spread via travel, given information on the prevalence of disease in the sending location and the rate of flow between two locations.

Although the paper builds on a published method, it should provide some additional detail about the method in the introductory sections. Specifically, in the section titled "Exportations" on page 3, the authors say that "the method assumes that the incubation period T_E and the infectious period T_I follow specific probability distributions."

More explanation of the modeling assumptions will allow potential users of this package to make an informed decision about whether it will be useful to them without going back to the original manuscript in which the method was described. In addition, the reuse of "T" to indicate the rate of travel in the paragraph above and the incubation and infectious periods is confusing and should be changed.

From reading the paper and looking at the github repository, it seems like it is completely up to the user to specify the distribution of the incubation and infectious periods. One potentially helpful addition to the package would be the addition of data with estimates of these quantities for different pathogens where available, along with citations/dois for the data source. This would make this more user-friendly and amenable to rapid response.

Is the rationale for developing the new software tool clearly explained? Yes

Is the description of the software tool technically sound? Partly

Are sufficient details of the code, methods and analysis (if applicable) provided to allow 
replication of the software development and its use by others?

Yes

Is sufficient information provided to allow interpretation of the expected output datasets and any results generated using the tool?

Yes

Are the conclusions about the tool and its performance adequately supported by the findings presented in the article?

Partly

Competing Interests: No competing interests were disclosed.

I confirm that I have read this submission and believe that I have an appropriate level of expertise to confirm that it is of an acceptable scientific standard, however I have significant reservations, as outlined above.

Author Response 24 Jul 2019

Paula Moraga, Lancaster University, Lancaster, UK

Thank you for your helpful and insightful comments. Please find our responses below.

1. Thank you for pointing out the choice of incubation and infectious period distributions needs clarification. In Section "Exportations" we have mentioned we assume that the incubation period and the infectious period are random variables, with associated probability distributions that are disease-specific. We have also cited the papers we used to choice the incubation and infectious period distributions of our yellow fever example.

2. Thank you for the notation review. Now we have changed the notation of the incubation period T_E by D_E, and infectious period T_I by D_I, where letter D stands for duration. Now letter $\mathrm{T}$ is only used for travelers.

3. We agree on the importance of the choice distributions of incubation and infectious periods. In Section "Arguments of the estimate_risk_spread() function", we have added that we can define these distributions using random generation functions of distributions that are implemented in R. However, we decided not to provide data for incubation and infectious periods of other pathogens since we expect users to have some knowledge of the life history of the disease they want to apply it to. We have mentioned that users should consider the literature carefully before deciding on appropriate distributions, and we reference two review papers that may be useful.

Competing Interests: No competing interests were disclosed. 
(C) 2018 Ross N. This is an open access peer review report distributed under the terms of the Creative Commons Attribution License, which permits unrestricted use, distribution, and reproduction in any medium, provided the original work is properly cited.

\section{Noam Ross}

EcoHealth Alliance, New York City, NY, USA

The authors present and provide a tutorial to epiflows, an R package for calculating the risk of travel-related disease export from an epidemic area. It is a useful implementation of an algorithm, with associated visualization tools. It is technically sound though the scaffolding around the core algorithm is somewhat over-engineered.

Both the package design and paper description imply this package is designed for rapid risk assessment. My comments are primarily in regard to the clarity of the description and the usability of the package API in this context.

The 'global_vars()' function is a thin wrapper around R's 'options()' mechanism that obfuscates what is actually happening, and the name itself is somewhat confusing. (There are many different uses and abuses of global variables in R). It is difficult to see how this function improves over simply telling the user that default variables are defined by 'epiflows.vars` in R's options mechanism (epiflows.varnames might be clearer).

While it is mentioned that the 'epiflows` object inherits from `epicontacts`, it is not clear what this means and how it is relevant to the user. Given the epiflows object has different contents than the 'epicontacts` object, it should be explained. I see in the package vignette that subsetting methods are inherited, but given that the object contents are different, this should be demonstrated in the paper. Otherwise it is hard to see what the advantage of this object is at all, over simply passing data frames to the algorithm function.

The paper (as well as the code demonstration and vignette in the package), spends considerable time on the conversion of the partially processed data in 'YF_Brazil' to data appropriately structured to be used in the 'make_epiflows()' and 'estimate_risk_spread()' function. This is confusing and not very useful - most users will not have data in exactly the format of 'YF_Brazil', and it distracts from the description of the core package functionality. It would be far clearer to introduce and demonstrate the functions first using data in its ready-to-analyze form. If the authors wish to provide an example of an actual full workflow, they should start with actual raw source data from the the supplementary data. A useful addition would also be to describe possible sources of the data needed.

The distributions of incubation and infectious periods is important and glossed over rather quickly here. First, on page 3, the text reads, "The method assumes that the incubation period TE and the infectious period TI follow specific probability distributions." This is unclear, I think "disease-specific" would be clearer. Moreover, in the code tutorial, these distributions are simply assumed. It would make more sense to describe why such distributions are selected and the source of the parameters, e.g., "For yellow fever we choose these distributions based on clinical literature describing the disease course as $X$ to $Y$ days of incubation and $V$ to $Z$ days infectious period (Citation 1, Citation 2). Lognormal or Gamma distributions are typically used for these distributions..." 
It also confusing that both individuals traveling and incubation times are shown as $\mathrm{T}$ in the mathematical notation.

Is the rationale for developing the new software tool clearly explained?

Yes

Is the description of the software tool technically sound?

Partly

Are sufficient details of the code, methods and analysis (if applicable) provided to allow replication of the software development and its use by others?

Yes

Is sufficient information provided to allow interpretation of the expected output datasets and any results generated using the tool?

Yes

Are the conclusions about the tool and its performance adequately supported by the findings presented in the article?

Yes

Competing Interests: No competing interests were disclosed.

Reviewer Expertise: Disease ecology, disease modeling, R programming an package design.

I confirm that I have read this submission and believe that I have an appropriate level of expertise to confirm that it is of an acceptable scientific standard, however I have significant reservations, as outlined above.

Author Response 24 Jul 2019

Paula Moraga, Lancaster University, Lancaster, UK

Thank you for your thorough review and insightful comments. We address each comment separately below.

1. In response to the comment regarding the 'global_vars()' function, we think this is a fair point. The main advantage of the use of 'global_vars()' is giving the user a way to easily recover the 'epiflows.vars' option variables in the case that they made an error in specifying new variables (global_vars(reset $=$ TRUE)).

2. Thank you for bringing to our attention that the use of epicontacts needs an explanation. Because a flow of cases from one location to another can be thought of as a contact with a wider scope, the 'epiflows` object inherits from the 'epicontacts` object, where locations are stored in the "linelist" element and flows are stored in the "contacts" element (though the user does not need to interact with these elements by name). By building on the 
epicontacts object, we ensure that all the methods for sub-setting an object of class 'epicontacts' also applies to 'epiflows', reducing the maintenance effort. We have clarified this in Section "The epiflows object" of the manuscript.

3. In order to present an example that is as clear as possible for readers, we have modified the yellow fever example and now we do not describe datasets that do not have ready-toanalyze form. Now we start by describing the YF_flows and YF_locations objects which can be directly passed to the make_epiflows() function to create the Brazil_epiflows object.

4. Thank you for pointing out the importance of the distributions of incubation and infectious periods. In Section "Exportations" we have replaced "the method assumes that the incubation period T_E and the infectious period T_I follow specific probability distributions." by "We assume that the incubation period (D_E) and the infectious period (D_I) are random variables, with associated probability distributions that are diseasespecific." In Section "Arguments of the estimate_risk_spread() function", we have cited the papers we used to choose the incubation and infectious period distributions of the yellow fever example. We decided not to provide data for incubation and infectious periods of other pathogens since we expect users to have some knowledge of the life history of the disease they want to apply it to. We have also mentioned that users should consider the literature carefully before deciding on appropriate distributions, and we reference two review papers that may be useful.

5. Thank you for the notation review. Now we have changed the notation of the incubation period T_E by D_E, and infectious period T_I by D_I, where letter D stands for duration. Now letter $\mathrm{T}$ is only used for travelers.

Competing Interests: No competing interests were disclosed.

The benefits of publishing with F1000Research:

- Your article is published within days, with no editorial bias

- You can publish traditional articles, null/negative results, case reports, data notes and more

- The peer review process is transparent and collaborative

- Your article is indexed in PubMed after passing peer review

- Dedicated customer support at every stage

For pre-submission enquiries, contact research@f1000.com 